\title{
Protective Effects of Oral Administrated Ascorbic Acid against Oxidative Stress and Neuronal Damage after Cerebral Ischemia/Reperfusion in Diabetic Rats
}

\author{
Naohiro Iwata, Mari Okazaki, ${ }^{*}$ Shinya Kamiuchi, and Yasuhide Hibino \\ Laboratory of Immunobiochemistry, Department of Clinical Dietetics \& Human Nutrition, Faculty of Pharmaceutical Sciences, Josai \\ University, 1-1 Keyakidai, Sakado, Saitama 350-0295, Japan
}

(Received June 8, 2009; Accepted October 8, 2009; Published online October 26, 2009)

\begin{abstract}
Diabetes enhances oxidative stress and exacerbates acute ischemic injury. Previous studies have demonstrated that infarct volumes were greater in diabetic animals caused by a transient cerebral ischemia as compared with nondiabetic animals. Ascorbic acid (AA) as a naturally occurring major antioxidant is reported to be low in diabetic tissues. Therefore, we examined the effects of daily supplementation of AA on lipid peroxidation and the activity of antioxidant enzymes (superoxide dismutase, catalase and glutathione peroxidase) in streptozotocin (STZ)-induced diabetic rat brain. Additionally, we also investigated whether AA improves the exacerbation of neuronal damage induced by middle cerebral artery occlusion followed by reperfusion $(\mathrm{MCAO} / \mathrm{Re})$ in diabetic state. Type1-diabetes was induced in male Sprague Dawley rats by STZ $(60 \mathrm{mg} / \mathrm{kg}$ of intraperitoneal injection). Five weeks after STZinjection, the diabetic rats showed enhanced lipid peroxidation and impaired activity of antioxidant enzymes in the brain. Furthermore, the gene expression of glucose transporter GLUT1, which locates in the endothelial cells of the blood-brain-barrier and transports oxidized AA as the main source of AA supply to the brain, was downregulated in the diabetic brain. AA-supplemented $(100 \mathrm{mg} / \mathrm{kg}$ daily, 2 weeks $)$ diabetic rats showed normal levels of all these parameters. A diabetic state markedly aggravated MCAO/Re-induced neurological deficits and cerebral injury assessed by infarction volume. Treatment of AA remarkably improved both parameters in the diabetic rats. These results suggest that daily intake of AA relieves the exacerbation of cerebral ischemic injury in a diabetic state, which may be attributed to improvement of augmented oxidative stress.
\end{abstract}

Key words — ascorbic acid (vitamin C), diabetes, middle cerebral artery occlusion (MCAO), oxidative stress, glucose transporter 1 (GLUT1)

\section{INTRODUCTION}

Oxidative stress induced by excessive production of free radicals is considered to be crucial to cell injury in a variety of diseases. Diabetes mellitus, which increases physiological oxidative stress attributed to chronic hyperglycemia, is a major risk factor for atherosclerotic disease such as acute brain ischemia. ${ }^{1,2)}$ Diabetic patients have a higher risk of stroke compared with non-diabetic patients and they are more likely to have a poor prognosis and increased mortality after stroke. ${ }^{3,4)}$ Previous studies have also demonstrated that diabetes increases ox-

\footnotetext{
${ }^{*}$ To whom correspondence should be addressed: Laboratory of Immunobiochemistry, Department of Clinical Dietetics \& Human Nutrition, Faculty of Pharmaceutical Sciences, Josai University, 1-1 Keyakidai, Sakado, Saitama 350-0295, Japan. Tel.: +81-49-271-7267; Fax: +81-49-271-7284; E-mail: okazaki@josai.ac.jp
}

idative stress in the brain ${ }^{5)}$ and aggravates cerebral ischemic injury in animal models. ${ }^{6-8)}$ In particular, hyperglycemia in diabetic state increases the formation of reactive oxygen species (ROS) after reperfusion of blood flow. ${ }^{9,10)}$ Treatment of natural antioxidants such as rutin, ${ }^{11)}$ a polyphenolic flavonoid, red wine, ${ }^{12)}$ plant extracts, ${ }^{13,14)}$ etc. has been reported to improve cerebral damage and dysfunction in diabetic state. Taking these findings together, it is supposed that enhanced oxidative stress in the brain induced by diabetic hyperglycemia contributes to the exacerbation of brain injury caused by transient ischemia.

Naturally occurring major antioxidant ascorbic acid (AA) is essential for scavenging free radicals in both plasma and tissues. It has been reported that the concentration of AA sensitively reflects oxidative stress in animal tissues. ${ }^{15)}$ There is evidence of lower levels of the concentrations of $\mathrm{AA}$ in the 
plasma, liver and kidney of diabetic animals and humans. ${ }^{16,17)}$ The decrease in AA of most tissues may be due to its enhanced consumption by elevated oxidative stress in the diabetic state. Indeed, AA supplementation prevents the development of diabetic complications associated with a decrease in the levels of hyperglycemia, hyperlipidemia, hyperketonemia, ${ }^{18)}$ and lipid peroxidation in the liver and muscle ${ }^{19)}$ of diabetic animals. Meanwhile, change in cerebral AA level in diabetic state and its cerebroprotective effects have not been wellestablished. ${ }^{20,21)}$ It has been reported that even a high dose of AA has no acute protective effect on damage induced by transient cerebral ischemia, because AA is not transportable through the bloodbrain-barrier (BBB) in vivo. ${ }^{22)}$ Contrary to the report, AA is likely to be transported across the BBB slowly, whereas no measurable transport of AA across the $\mathrm{BBB}$ at a short time interval was detected. ${ }^{23)}$ Furthermore, several studies in vitro have shown that AA also has pro-oxidant properties, and induces lipid peroxidation and apoptosis in neuronal cells. $^{24-26)}$ Thus, it is unclear whether continuous oral administration of AA can protect against a transient cerebral ischemia in diabetic state.

In the present study, we examined the effect of oral administration for 2 weeks of a comparatively low dose of AA ( $100 \mathrm{mg} / \mathrm{kg}$ per day) on the serum level of hydroperoxide as a marker of total oxidative stress and biological antioxidant potential in streptozotocin (STZ)-diabetic rats. The level of thiobarbituric acid reactive substances (TBARS) as a lipid peroxidation marker, and activity of antioxidant enzymes, i.e., superoxide dismutase (SOD), catalase (CAT) and glutathione peroxidase (GPx) in the brain were also evaluated. Furthermore, we examined whether AA protects diabetic rats against the transient focal cerebral ischemia using a model of middle cerebral artery occlusion followed by reperfusion (MCAO/Re). Additionally, the expression level of mRNA of glucose transporter GLUT1 which locates in the endothelial cells of $\mathrm{BBB}$ and transports oxidized AA (dehydroascorbic acid: DHA) as a main source of AA supply to a brain were examined. We found that the daily supplementation of AA attenuated oxidative stress both in plasma and the brain, and improved cerebral injury induced by transient ischemia in diabetic rats.

\section{MATERIALS AND METHODS}

Experimental Diabetic Animals — Male Sprague Dawley rats (4-week old, weight 120-140 g; Tokyo Experimental Animal Co., Ltd., Tokyo, Japan) were used and housed two to a cage in a temperature-controlled environment $\left(23 \pm 0.5^{\circ} \mathrm{C}\right)$ with a cycle of $12 \mathrm{hr}$ light and $12 \mathrm{hr}$ dark. The rats were given rodent chow and water ad libitum. Animal care and the surgical procedure were performed in accordance with guidelines approved by the $\mathrm{Na}$ tional Institutes of Health and the Josai University Animal Investigation Committee. A diabetic state was induced in the rats by a single intraperitoneal injection of STZ $(60 \mathrm{mg} / \mathrm{kg})$ dissolved in $0.1 \mathrm{mM}$ sodium citrate ( $\mathrm{pH} 4.5$ ) (diabetic group), while the rats of the non-diabetic group were injected with buffer only. ${ }^{6}$ ) Seven days after the STZ-injection, a blood sample was collected by tail-vein paracentesis, and then plasma glucose was determined using a glucose analyzer (Ascensia, Bayer Medical Co., Ltd., Land Nordrhein-Westfalen, Germany). Diabetes was defined by a blood glucose level greater than $300 \mathrm{mg} / \mathrm{dl}$. Then, the diabetic and non-diabetic groups were divided into two groups respectively and were housed for an additional 6 weeks until stroke was induced by MCAO. AA $(100 \mathrm{mg} / \mathrm{kg}$; AA supplemented group) or distilled water (control group) was administrated orally once daily for the last 2 weeks.

Measurement of Systemic Oxidative Stress The serum levels of hydroperoxide as a marker of total oxidative stress were measured by active oxygen free radical autoanalyzer (Free Radical Elective Evaluator: F.R.E.E.) using Diacron-Reactive Oxygen Metabolites (d-ROMs) test kit as previously reported. ${ }^{27)}$ The results of the d-ROMs test were expressed in arbitrary units called "Carratelli units" (CARR U), where 1 CARR U corresponds to $0.08 \mathrm{mg} / 100 \mathrm{ml} \mathrm{H}_{2} \mathrm{O}_{2} .{ }^{27)}$ Plasma antioxidant capacity was also detected using Biological Antioxidant Potential (BAP) test kits. F.R.E.E. and the kits were purchased from Diacron International s.r.l. (Grosseto, Italy).

Preparation of Brain Extract — After a 2-week supplementation period, the rats were anesthetized deeply with diethyl ether, and then decapitated for measurement of lipid peroxidation and activity of antioxidant enzymes. The brain was removed quickly and was separated into cortex, striatum + hippocampus, cerebellum, and brainstem. 
Estimation of Lipid Peroxidation — The lipid peroxide content in the brain tissues was assayed in the form of TBARS by the method of Ohkawa et al. ${ }^{6,28)}$ Briefly, the brain tissues $(10 \% \mathrm{w} / \mathrm{v})$ were homogenized in chilled potassium chloride solution $(1.15 \%)$. This solution included protease inhibitors; $1 \mathrm{mg} / \mathrm{ml}$ Pepstatin A, $1 \mathrm{mg} / \mathrm{ml}$ Leupeptin, $10 \mathrm{mM}$ phenylmethylsulfonyl fluoride (Wako Pure Chemicals, Tokyo, Japan), and $1 \mu \mathrm{l} / \mathrm{ml}$ Trasylol (Bayer HealthCare AG, Land Nordrhein-Westfalen, Germany). Briefly, the reaction mixture consisted of $0.2 \mathrm{ml}$ of $8.1 \%$ sodium dodecyl sulphate, $0.05 \mathrm{ml}$ of $20 \%$ acetic acid buffer solution (pH 3.5$), 1.5 \mathrm{ml}$ of $0.8 \%$ acetic acid solution of butylated hydroxytoluene (BHT), $1.5 \mathrm{ml}$ of $0.8 \%$ aqueous solution of 1,3-diethyl-2-thiobarbituric acid (Sigma-Aldrich, St. Louis, MO, U.S.A.), $0.7 \mathrm{ml}$ of distilled water was added sequentially to $0.1 \mathrm{ml}$ of $10 \%(\mathrm{w} / \mathrm{v})$ tissue-homogenate in a test tube with a cap, and then the mixture was stirred and cooled to room temperature. The mixture was heated at $95^{\circ} \mathrm{C}$ for $60 \mathrm{~min}$. After cooling, $1.0 \mathrm{ml}$ of distilled water and $5.0 \mathrm{ml}$ of the mixture of $n$-butanol (Nacalai Tesque, Tokyo, Japan) and pyridine (15:1, v/v, Wako Pure Chemicals) was added and mixed. Then, the mixture was centrifuged at $3000 \mathrm{rpm}$ for $10 \mathrm{~min}$ at room temperature. The organic layer was removed and its absorbance was measured at $532 \mathrm{~nm}$. TBARS were quantified using an extinction coefficient of $\left(\mathrm{A}-\mathrm{A}_{0}\right) / 156000 \times 5.8 / 10^{3} \times 10^{2} / 3 \times 10^{6}$ and expressed as nmol of TBARS per mg protein. The tissue protein content of each sample was estimated using the Lowry method of protein assay.

CAT — CAT activity in the brain tissues was assayed by the method of Tirkey et al. ${ }^{6,29}$ The assay mixture consisted of $1.94 \mathrm{ml}$ phosphate buffer (50 mM, pH 7.0), $1.0 \mathrm{ml}$ hydrogen peroxide $(59 \mathrm{mM})$, and $0.06 \mathrm{ml}$ brain tissue homogenate in a total volume of $3.0 \mathrm{ml}$. Changes in absorbance were recorded at $240 \mathrm{~nm}$. CAT activity was calculated in terms of $\mathrm{nM} \mathrm{H}_{2} \mathrm{O}_{2}$ consumed/min per mg protein.

SOD — SOD activity in the brain tissues was measured using SOD Assay Kit-WST (Dojindo Laboratories, Tokyo, Japan), which is based on the principle in which xanthine reacts with xanthine oxidase to generate superoxide anion radicals reacting with WST-1 to form a formazan dye. Brain tissues $(20 \%, \mathrm{w} / \mathrm{v})$ homogenized in $10 \mathrm{mmol} / \mathrm{l}$ Tris- $\mathrm{HCl}$ buffer solution ( $\mathrm{pH} 7.4$ ) containing $0.25 \mathrm{~mol} / \mathrm{l}$ of sucrose and $1 \mathrm{mmol} / \mathrm{l}$ EDTA were used to the assay. ${ }^{6}$ ) GPx — GPx activity in the brain tissues was measured according to the procedure previously described. ${ }^{30)}$ The reaction mixture consisted of $1.44 \mathrm{ml}$ phosphate buffer (50 mM, pH 7.0), $0.1 \mathrm{ml}$ of EDTA ( $1 \mathrm{mM}), 0.1 \mathrm{ml}$ of sodium azide $(1 \mathrm{mM})$, $0.05 \mathrm{ml}$ of glutathione reductase $(1 \mathrm{U} / \mathrm{ml}), 0.1 \mathrm{ml}$ of glutathione $(1 \mathrm{mM}), 0.1 \mathrm{ml}$ of NADPH $(0.2 \mathrm{mM})$, $0.01 \mathrm{ml}$ of hydrogen peroxide $(0.25 \mathrm{mM})$ and $0.1 \mathrm{ml}$ of post mitochondrial supernatant $(10 \%, w / v)$ in a final volume of $2.0 \mathrm{ml}$. The disappearance of $\mathrm{NADPH}$ at $340 \mathrm{~nm}$ was detected at room temperature. The enzyme activity was represented as $\mathrm{nM}$ NADPH oxidized/min per $\mathrm{mg}$ protein.

Evaluation of GLUT1 Gene Expression by RealTime PCR Analysis — Gene expression analysis of GLUT1 was conducted using quantitative realtime PCR as described earlier. ${ }^{31)}$ Total RNA was extracted from the ipsilateral cortex with RNeasy Mini Kit (QIAGEN, Hilden, Germany) according to the manufacturer's instructions. Total RNA $(0.5 \mu \mathrm{g})$ was reverse-transcribed with oligo dT and random hexamer primers using reverse transcriptase (PrimeScript $^{\mathrm{TM}}$ RT Enzyme Mix I, Takara RNA PCR Kit, Takara Biomedicals, Otu, Shiga, Japan). Real-time PCR was performed with $10 \mathrm{ng}$ of cDNA and a pair of GLUT1 gene specific primers (Takara Biomedicals) added to the SYBR Premix EX Taq (Takara Biomedicals) and subjected to PCR amplification in iCycler iQ Real-Time Detection System ( 1 cycle at $95^{\circ} \mathrm{C}$ for $10 \mathrm{sec}$, and 50 cycles at $95^{\circ} \mathrm{C}$ for $5 \mathrm{sec}$ and $60^{\circ} \mathrm{C}$ for $34 \mathrm{sec}$; Bio-Rad Laboratories, Inc., Hercules, CA, U.S.A.). The expression of $\beta$-actin was used to normalize cDNA levels. The PCR products were analyzed by a melting curve to ascertain the specificity of amplification.

MCAO and Reperfusion- MCAO was performed using a standard intraluminal procedure as previously described. ${ }^{6,32)}$ Rats were anesthetized with halothane (induction: $4 \%$, maintenance: $1.5 \%$ ) in $30 \%$ oxygen with a facemask. After a midline incision at the neck, the right common carotid artery was exfoliated under an operating microscope. All branches of external carotid artery were ligated, and isolated. The tips of the 4-0 surgical nylon monofilament were rounded by flame heating, and the tip was inserted up through the internal carotid artery. When a small resistance was felt, insertion was stopped. Cerebral blood flow detected laser by Doppler flowmetry (ATBF-LC1, Unique Medical Co., Ltd., Tokyo, Japan) was reduced to $49.6 \pm 5.4 \%$ $(n=7)$ of baseline after MCAO in non-diabetic and diabetic rats. At $2 \mathrm{hr}$ of the occlusion, the filament was withdrawn to allow for reperfusion. The distance from bifurcation of the common carotid artery 
to the tip of the suture was approximately $20 \mathrm{~mm}$ in all rats. Successful occlusion of the right MCA was achieved when the left forelimb was paretic after the nylon filament was introduced. The animals were permitted to recover from the anesthesia at room temperature. Rectal temperature was maintained at $37^{\circ} \mathrm{C}$ with a heat lamp and a heating pad during the operation. All the rats were killed at $24 \mathrm{hr}$ of reperfusion. The sham operation occurs with the same manipulation without introduction of the monofilament.

Neurological Evaluation — Post-ischemic neurological deficits were evaluated after $2 \mathrm{hr}$ MCAO, and at $24 \mathrm{hr}$ of reperfusion on a 5-point scale as described as follows: grade 0: no deficit, grade 1 : failure to extend right forepaw fully, grade 2 : spontaneous circling or walking to contralateral side, grade 3 : walking only when stimulated, grade $4:$ unresponsive to stimulation and a depressed level of consciousness, grade $5:$ death. ${ }^{6)}$ Animals that did not show neurological deficits were excluded from the study.

Infarct and Edema Assessment - At $24 \mathrm{hr}$ of reperfusion, the animals were deeply anesthetized with diethyl ether and decapitated. The brain was removed and cut into four $2 \mathrm{~mm}$ coronal sections by use of a rat brain matrix, and stained with $2 \%$ 2,3,5triphenyltetrazolium chloride (TTC; Wako Pure Chemicals) at $37^{\circ} \mathrm{C}$ for $15 \mathrm{~min}$. The coronal slices were fixed in $10 \%$ formaldehyde for photography. And then, infarct areas were determined by using image analysis system (Scion Image 1.62), and were added to obtain the infarct volumes per brain. Corrected infarct volume $(\%)=[$ left hemisphere volume - (right hemisphere volume - the infarct volume)] / left hemisphere volume $\times 100$. Edema in the ischemic hemisphere was also calculated: edema $(\%)=($ right hemisphere volume - the infarct volume) / left hemisphere volume. ${ }^{6}$ )

Statistical Analysis — Data are represented as mean \pm S.D. Statistical analysis for physiological and biochemical parameters, GLUT1 mRNA levels, and area of cerebral damage was performed with a one-way analysis of variance (ANOVA) followed by post hoc Tukey's multiple-comparison test. Neurological deficit scores were analyzed by KruskalWallis test followed by the Mann-Whitney $U$ test. In all cases a $p$-value of $<0.05$ was taken as the level of significance.

\section{RESULTS}

\section{Physiological Characteristic Parameter}

The possible effect of AA supplementation on the body weight and non-fasting blood glucose level were examined in the non-diabetic and diabetic groups. Table 1 shows that the control diabetic group had typical characteristics of type $1 \mathrm{di}$ abetes such as a decrease in the body weight and hyperglycemia compared with the non-diabetic rats, which were similar to previous reports. ${ }^{5,17)}$ Daily supplementation of AA for 2 weeks did not show a significant effect on either body weight loss or hyperglycemia in the diabetic rats.

\section{Oxidative Stress and Antioxidant Activities in Plasma and Brain}

The serum levels of hydroperoxide as a marker of systemic oxidative stress in the non-diabetic and diabetic groups were measured using the d-ROMs test (Fig. $1 \mathrm{~A}$ ). The oxidative stress in the control diabetic group $(252.8 \pm 32.9$ U.CARR $)$ significantly increased to about 2 times that of the control non-diabetic group $(131.0 \pm 7.0$ U.CARR $)$. In contrast, this parameter was decreased in the AA supplemented diabetic group to the normal level $(137.3 \pm 32.1$ U.CARR). The antioxidant ability in the plasma measured by BAP test was significantly attenuated in the control diabetic rats $(1475.7 \pm 67.1)$ in comparison with the non-diabetic rats $(1875.4 \pm$ 156.1, Fig. 1 B). This parameter of the AA supplemented diabetic group $(1454.7 \pm 101.9)$ was not significantly different with the control diabetic group. The control diabetic rats had an elevated level of TBARS (Fig. 2) and decreased activity of antioxidant enzymes CAT, SOD, and GPx (Fig. 3) in the brain tissue compared with the non-diabetic rats. Supplementation of AA in diabetic rats showed a reversal of these parameters to near normal levels in most region of the brain.

Table 1. Effect of Orally Supplementation of AA for Two Weeks on Body Weight and Blood Glucose in Experimental Groups of Rats

\begin{tabular}{lcc}
\hline \hline Group & $\begin{array}{c}\text { Body weight } \\
(\mathrm{g})\end{array}$ & $\begin{array}{c}\text { Blood glucose } \\
(\mathrm{mg} / \mathrm{dl})\end{array}$ \\
\hline non-DM+H ${ }_{2} \mathrm{O}$ & $402.3 \pm 28.1$ & $137.6 \pm 17.1$ \\
non-DM+AA & $411.4 \pm 20.8$ & $146.1 \pm 11.5$ \\
$\mathrm{DM}+\mathrm{H}_{2} \mathrm{O}$ & $262.1 \pm 67.4^{a)}$ & $744.3 \pm 81.3^{a)}$ \\
$\mathrm{DM}+\mathrm{AA}$ & $304.3 \pm 48.7^{a)}$ & $607.2 \pm 88.1^{a)}$ \\
\hline
\end{tabular}

Values are given as mean \pm S.D. a) $p<0.01$ vs. non-DM $+\mathrm{H}_{2} \mathrm{O}$ group. $n=10$. 
(A) d-ROMs test

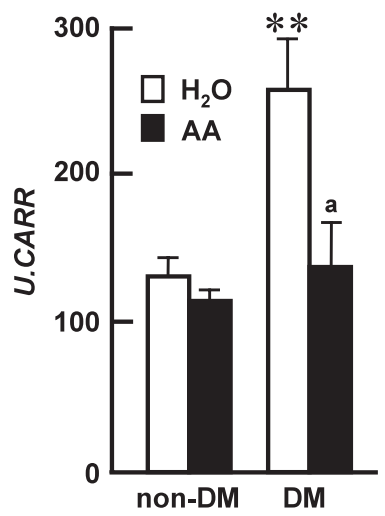

(B) BAP test

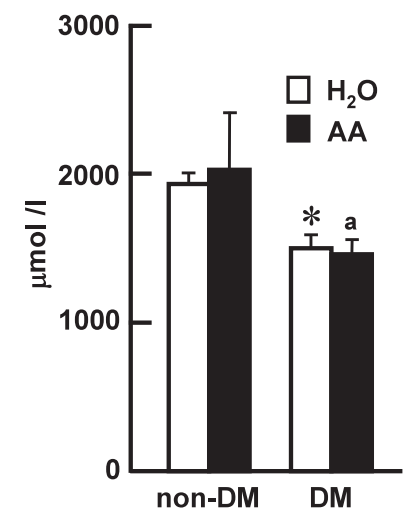

Fig. 1. Effects of AA Supplementation on Total Oxidative Stress (A) and Antioxidative Capacity (B) in Plasma of the Non-diabetic (Non-DM) and the Diabetic (DM) Rats Determined by d-ROMs Test and BAP Test, Respectively

Data are mean \pm S.D. of 6-7 rats per group. ${ }^{* * * *} p<0.05,0.01$ for statistically significant compared to distilled water $\left(\mathrm{H}_{2} \mathrm{O}\right)$-administered non-DM group. ${ }^{a} p<0.01$ compared to the $\mathrm{H}_{2} \mathrm{O}$-administered DM group.

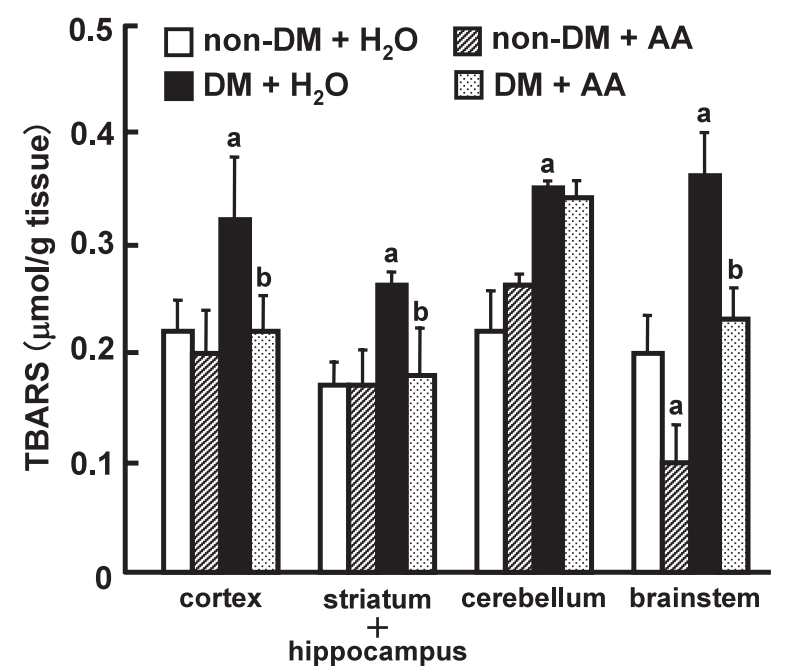

Fig. 2. Effect of AA Supplementation on the Level of Lipid Peroxidation in the Brain of the Non-DM and the DM Rats Determined by TBARS Assay

Data are mean \pm S.D. of 6-7 rats per group. ${ }^{a} p<0.01$ for statistically significant compared to distilled water $\left(\mathrm{H}_{2} \mathrm{O}\right)$-administered nonDM group. ${ }^{\mathrm{b}} p<0.01$ compared to the $\mathrm{H}_{2} \mathrm{O}$-administered DM group.

\section{Expression of GLUT1 in Cortex}

The gene expression of GLUT1 in the brain was quantified using real-time PCR by normalization with $\beta$-actin expression (Fig. 4). Diabetic state significantly down-regulated the expression of GLUT1 in the cerebral cortex as compared with the nondiabetic rats. Supplementation with AA increased the expression of GLUT1 both in the diabetic and non-diabetic rats and made up the difference between the two groups.

\section{Neurological Deficits and Infarct Volume after Transient MCAO with Reperfusion}

MCAO for $2 \mathrm{hr}$ in non-diabetic rats resulted in moderate neurological deficits, and the neurological evaluation value was increased after $2 \mathrm{hr}$ MCAO (Fig. $5 \mathrm{~A}$ ). In particular, the following $24 \mathrm{hr}$ reperfusion markedly exacerbated the neurological deficits. In the diabetic rats subjected to the transient MCAO, severe neurological dysfunction was observed compared to the non-diabetic rats (Fig. 5 B). Furthermore, the value was remarkably increased by reperfusion in diabetes. On the other hand, AA supplementation showed a significant improvement effect for the neurological dysfunction caused by MCAO with reperfusion in diabetic state.

Figure 6 shows TTC-stained coronal brain sections from representative rats at $24 \mathrm{hr}$ reperfusion of the four groups. In the sham operated animals, there was no apparent damage in any brain region. The infarct area in the non-diabetic rats after MCAO with $24 \mathrm{hr}$ reperfusion was extended to corpus striatum and cortex, whereas it was significantly decreased by AA supplementation (Fig. 7 A). The control diabetic rats have a large infarction extended to the whole thalamus and cortex. The lesion volume in the diabetic rats was significantly increased about 2.5 times compared with the non-diabetic rats. AA supplementation in the diabetic animals remarkably decreased the infarction volume, in which the lesion was determined only in the cortex and part of the thalamus. In addition, AA supplementation also reduced the edema exacerbated by diabetes (Fig. 7 B).

\section{DISCUSSION}

The findings of the present study indicate that the chronic oral supplementation of AA improves the antioxidant status of the diabetic rat brain by decreasing lipid peroxidative products and restoring impaired antioxidant enzyme activity. Furthermore, we demonstrated for the first time that orally supplemented AA alleviates the exacerbation in cerebral ischemic injury caused by MCAO with reperfusion in the STZ-induced diabetic rats. In diabetic state, "glucose toxicity" caused by augmentation of intracellular glucose oxidation process and nonenzymatic glycation of protein molecules leads to over production of ROS and damage of neurons and en- 
(A)

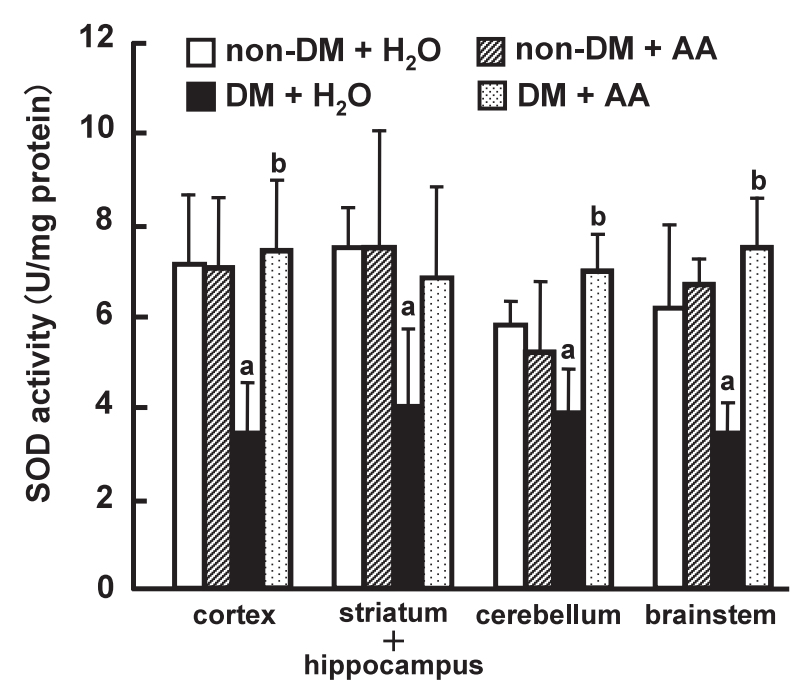

(B)

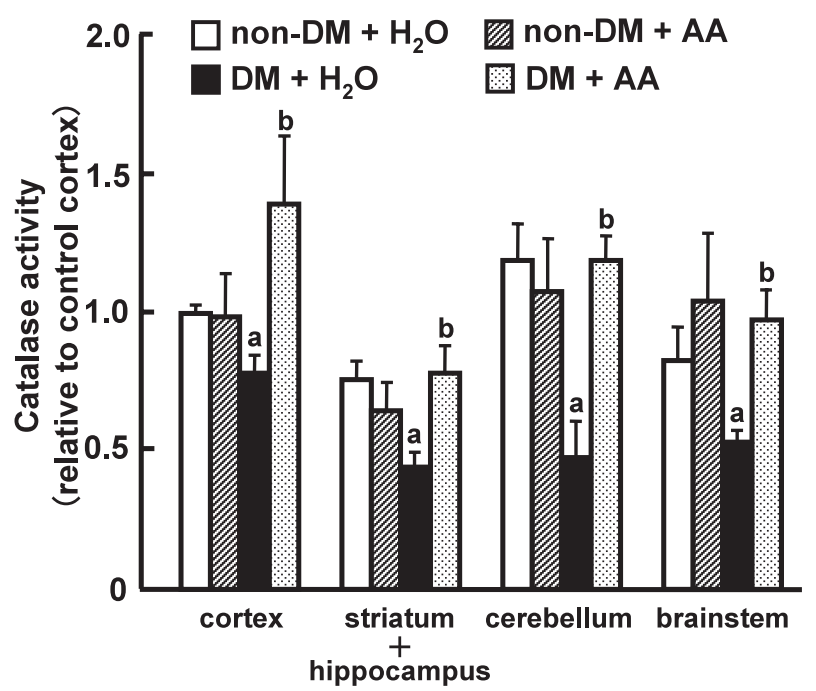

(C)

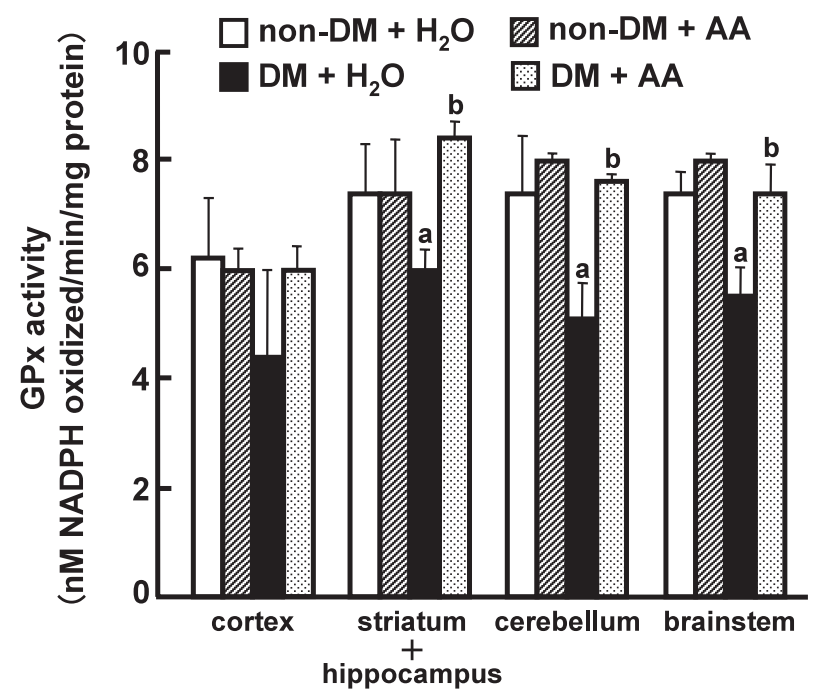

Fig. 3. Effect of AA Supplementation on the Activity of SOD (A), CAT (B), and GPx (C) in the Non-DM and the DM Rat Brain Data are mean \pm S.D. of $6-7$ rats per group. ${ }^{a} p<0.01$ for statistically significant compared to the distilled water $\left(\mathrm{H}_{2} \mathrm{O}\right)$-administered non-DM group. ${ }^{\mathrm{b}} p<0.01$ compared to the $\mathrm{H}_{2} \mathrm{O}$-administered DM group.

dothelial cells. ${ }^{1)}$ Augmented oxidative stress such as increased ROS production, augmented lipid peroxidation, and reduction of antioxidants has been indicated in the brain, kidney, pancreas, and liver of STZ-induced diabetic rats. ${ }^{5)}$ Additionally, impairment of antioxidant enzymes, caused by nonenzymatic glycation of protein molecules, increases lipid peroxidation in the brain of diabetic animals has been indicated. ${ }^{1,33)}$ In agreement with these reports, we observed that the level of lipid peroxidation marker TBARS was elevated and the activity of antioxidant enzymes CAT, SOD, and GPx was decreased in the all parts of the diabetic rat brain. The augmentation of lipid peroxidation in the brain might be a reflection of decreased activities of the antioxidant enzymes as defense systems.

AA plays a role in many biological processes including free radical scavenging and protection of lipid membranes as well as hormone synthesis and homeostasis. It is conceivable that the enhanced oxidative stress in diabetes consumes AA, and that the resulting diminution in AA leads to further enhancement of radical reactions and other dysfunctions in the brain. In fact, there is experimental ev- 


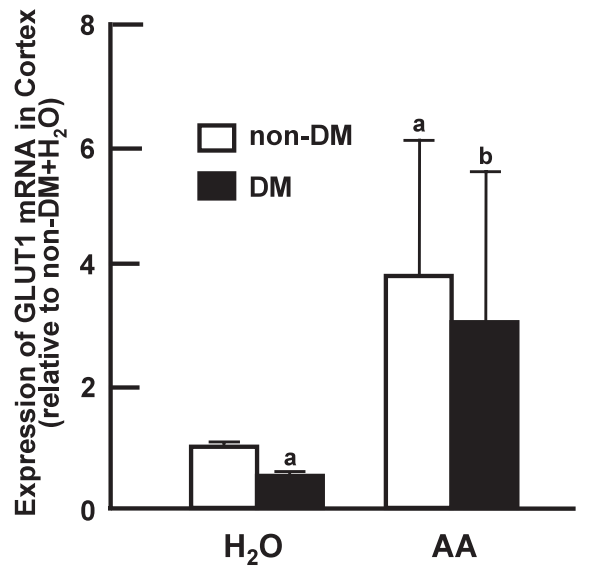

Fig. 4. Effect of AA Supplementation on Expression of GLUT1 mRNA in the Non-DM and the DM Rat Cortex

Data are mean \pm S.D. of $6-7$ rats per group. ${ }^{a} p<0.01$ for statistically significant compared to the distilled water $\left(\mathrm{H}_{2} \mathrm{O}\right)$-administered non-DM group. ${ }^{\mathrm{b}} p<0.01$ compared to the $\mathrm{H}_{2} \mathrm{O}$-administered DM group.

idence of lower levels of AA concentrations in the plasma, brain, and other tissues of the diabetic rats 8 weeks after intraperitoneal injection of STZ. ${ }^{18)} \mathrm{We}$ observed that the oral supplementation of AA prevented the lipid peroxidation and restored the impaired activity of the antioxidant enzymes, which could be as a result of improved antioxidants' status in the diabetic brain. AA had little effect on the elevated blood glucose level in the diabetic rats, confirming that the cerebroprotective effect of AA could be due to its antioxidant activity.

There is a relatively high concentration of AA in the brain. Previously, various types of AArelated transporters such as sodium-vitamin $\mathrm{C}$ cotransporter $^{34)}$ and GLUT ${ }^{35)}$ have been identified. A recent study has reported that the transport of DHA to the brain through a facilitative glucose transporter GLUT1 in endothelial cells of BBB, which was the main source of AA supply to the brain, is obstructed by hyperglycemia in STZ-induced diabetic state because of competitive inhibition of dextroglucose Dglucose. $^{36)}$ It has also been reported that diabetic hyperglycemia alters GLUT1 expression and activity in the brain. ${ }^{37-39)}$ Our study demonstrated that the expression level of GLUT1 mRNA in the cortex of diabetic rats was down-regulated. Thus, it can be supposed that not only the increased consumption of AA but suppression of DHA transport may be caused in the diabetic brain.

On the other hand, the cerebroprotective effect of AA supplementation is controversial, whereas beneficial effects of DHA administration for neuronal oxidative stress and inflammation seem to be
(A) non-DM

(B) DM
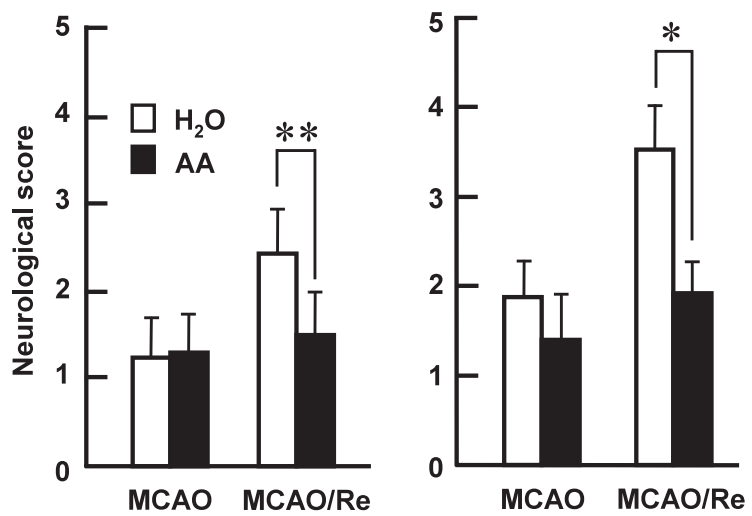

Fig. 5. Effect of AA Supplementation on Neurological Deficits Determined $2 \mathrm{hr}$ after MCAO and $24 \mathrm{hr}$ at Reperfusion (MCAO/Re) in Non-DM (A) and DM rats (B)

Data are mean \pm S.D. of 6-7 rats per group. ${ }^{* * *} p<0.05,0.01$ for statistically significant compared to individual distilled water $\left(\mathrm{H}_{2} \mathrm{O}\right)$ administered groups.

estimated. ${ }^{40-42)}$ Ahn et al. ${ }^{5)}$ revealed that AA supplementation has little effect against the elevated oxidative stress in the STZ-diabetic brain, whereas it is efficacious in other tissues. Previous studies do not support a role for an acute effect of supplementation of AA in normal animals, because AA is not transportable through the BBB in a short time period; even a high dose of AA $(500 \mathrm{mg} / \mathrm{kg}$ of intravenous administration) has no acute protective effect on the damage induced by transient cerebral ischemia in mice. ${ }^{40)}$ Agus et al. ${ }^{23)}$ indicated that a small amount of AA was slowly transported across the $\mathrm{BBB}$ in normal mice and rats by in vivo transport studies using isotope-labeled AA. In the present study, we observed that the oral supplementation of a relatively low dose $(100 \mathrm{mg} / \mathrm{kg}$ per day) of AA shows the antioxidant effect in the brain of diabetic rats. The discrepancies among the reports including our result may be explained on the grounds that AA shows a biphasic effect as anti-oxidant and prooxidant and has an optimal dose for neuroprotection as well as experimental conditions such as the duration and stage of diabetes. ${ }^{24,43)}$ Chronic AA supplementation for 2 weeks also may contribute to efficacy in the transport and utilization of AA in the brain.

The plasma levels of oxidative stress determined by d-ROMs test decreased in the AA-treated diabetic rats. On the other hand, plasma antioxidant capacity estimated by BAP test was not affected by AA. AA might be consumed immediately to re- 


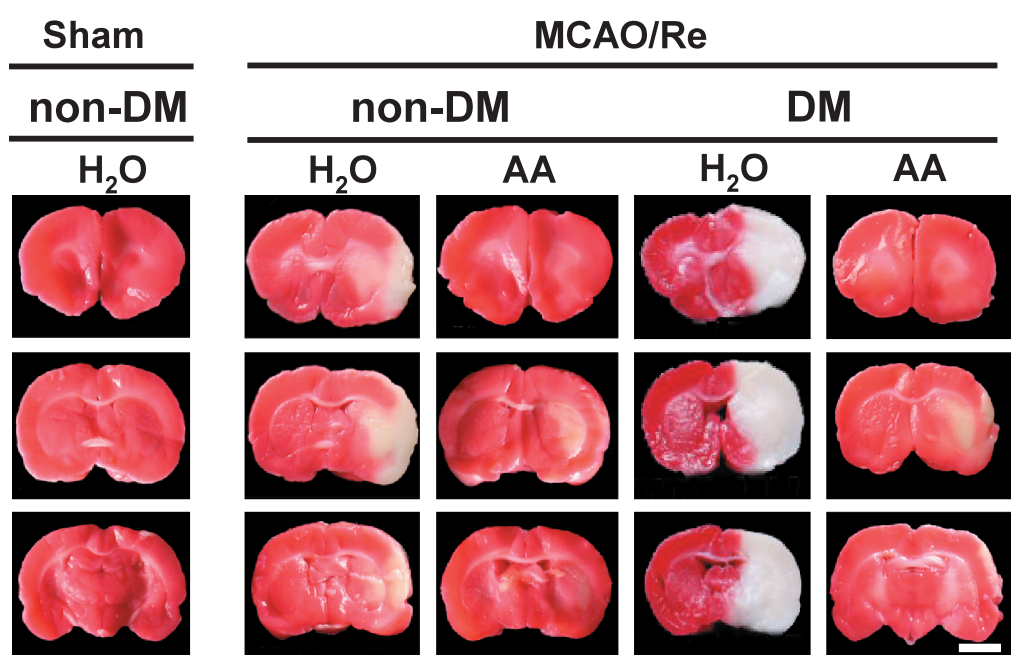

Fig. 6. Representative Data of TTC Staining from the Rats of Non-DM with Sham Operation Group, Distilled Water Administered $\left(\mathrm{H}_{2} \mathrm{O}\right)$ or AA Supplemented (AA) Non-DM or DM Group with MCAO followed by Reperfusion (MCAO/Re) AA $(100 \mathrm{mg} / \mathrm{kg}$ body weight) or water was administrated once daily for 2 weeks p.o. Horizontal bar: $3 \mathrm{~mm}$.

(A)

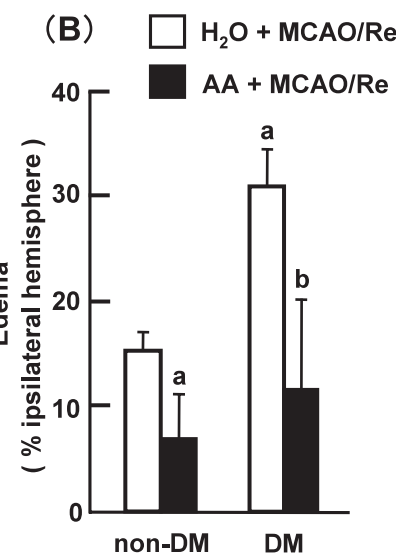

Fig. 7. Effect of AA Supplementation on Infarct Volume (A) and Edema (B) induced by MCAO/Re in Non-DM and DM Rats

Statistical significant reduction in total infarct volume and edema was observed in AA supplemented groups as compared to distilled water $\left(\mathrm{H}_{2} \mathrm{O}\right)$ - administered groups in both non-DM and DM rats. Data are presented as mean \pm S.D. $(n=6) .{ }^{\text {a }} p<0.05$ for statistically significant compared to $\mathrm{H}_{2} \mathrm{O}$-administered non-DM group. ${ }^{\mathrm{b}} \mathrm{p}<0.01$ compared to the $\mathrm{H}_{2} \mathrm{O}$-administered DM group.

duce excessive ROS produced under hyperglycemic condition, and it might contribute little to restoration of antioxidant capacity. Oxidation of AA to DHA is considered as a potentially important step in regulating AA entry into the brain, because AA is present in plasma mainly in its reduced form. ${ }^{44)}$ It is possible that AA tends to be oxidized to DHA under the elevated oxidative stress in the plasma of diabetic animals, which might overcome the competitive inhibition in BBB-transport caused by high concentration of glucose. In addition, it is commonly assumed that a high concentration of DHA could be produced in vivo by impaired cells and inflammatory cells. We found that the chronic supplementation of AA for 2 weeks reversed the decreased level of GLUT1 expression in the diabetic brain to the normal level, which may contribute to the recruitment of AA into the brain and correction of AA homeostasis. However, the precise process by which the orally supplemented AA exerts its effect in the brain remains unclear, hence further studies should be undertaken to reveal the change in the transport and metabolism of AA in the diabetic brain.

Oxidative stress is implicated also in the pathogenesis of the transient cerebral ischemic injury. Cerebroprotective effects of antioxidants increasing free radical degradation, inhibitors of free radical production, and free radical scavengers have been estimated in animal models. In this study, we observed that the diabetic model had a significantly greater lesion volume and edema by MCAO following reperfusion when compared to normal as previously reported. ${ }^{6}$ There are a number of factors that may explain the severe symptom of brain ischemia in diabetes; however the mechanisms exacerbating the ischemic injury of the diabetic brain have not been completely elucidated. As mentioned above, the hyperglycemia-induced dysfunction of enzymatic antioxidant defense causes accumulation of ROS, leading to lipid peroxidation and neuronal apoptosis. Actually, it has been demonstrated that apoptosis of cells in the penumbral region was enhanced in diabetic animals correlated with serum glucose. ${ }^{7)}$ ROS is also considered to 
induce matrix metalloproteinase and inflammatory cytokines via activation of nuclear factor (NF)- $\kappa \mathrm{B}$, leading to BBB disruption and edema formation. ${ }^{45}$ ) Furthermore, the ischemic cerebral injury in the diabetic state may be aggravated by acidosis, activation of aldose reductase and $\mathrm{NAD}(\mathrm{P}) \mathrm{H}$ oxidase, enhanced production of advanced glycation endproducts, protein kinase $\mathrm{C}$ activation induced by excessive $\mathrm{Ca}^{2+}$ influx, etc. We observed that exacerbation of the damage in the brain of diabetic rats mainly occurred during the reperfusion phase. Because the hyperperfusion of blood flow often associated with reperfusion locally produces a large amount of ROS and generates free radical chain reactions, it is conceivable that increased oxidative stress in the diabetic state enhances these processes. Our results suggest that the oral supplementation of AA protects neuronal cells and endothelial cells through the suppression of diabetic oxidative stress resulting in alleviating the extensive cerebral infarction and edema of the diabetic brain.

In conclusion, in this study, we found that chronic supplementation of a relatively low dose of AA protects STZ-induced diabetic rats against total oxidative stress and an exacerbation in cerebral ischemic injury. AA is expected to act as a scavenger of free radicals contributing to mitigation of oxidative neuronal injury in the diabetic brain. Furthermore, we found that the expression level of GLUT1 was down-regulated in the diabetic cortex, and supplementation of AA reversed the decreased level of GLUT1 expression in the diabetic brain to normal, which might contribute to the recruitment of AA into the brain and its cerebroprotective effects.

Acknowledgements We would like to thank Drs. Yasushi Nakada and Masaya Nakagawa (Toyama Chemical Co., Ltd., Tokyo, Japan) for the MCAO technique. This study was supported by Grants-inAid for Scientific Research from the Japan Society for the Promotion of Science (No. 16790450 and 19590700).

\section{REFERENCES}

1) Baynes, J. W. (1991) Role of oxidative stress in development of complications in diabetes. Diabetes, 40, 405-412.

2) Stephens, J. W., Khanolkar, M. P. and Bain, S. C. (2009) The biological relevance and measurement of plasma markers of oxidative stress in diabetes and cardiovascular disease. Atherosclerosis, 202, 321329.

3) Biller, J. and Love, B. B. (1993) Diabetes and stroke. Med. Clin. North Am., 77, 95-110.

4) Vinik, A. and Flemmer, M. J. (2002) Diabetes and macrovascular disease. J. Diabetes Complications, 16, 235-245.

5) Ahn, T., Yun, C. H. and Oh, D. B. (2006) Tissuespecific effect of ascorbic acid supplementation on the expression of cytochrome P450 2E1 and oxidative stress in streptozotocin-induced diabetic rats. Toxicol. Lett., 166, 27-36.

6) Iwata, N., Okazaki, M., Kasahara, C., Kamiuchi, S., Suzuki, F., Iizuka, H. and Hibino, Y. (2008) Protective effects of a water-soluble extract from culture medium of Ganoderma lucidum mycelia against neuronal damage after cerebral ischemia/reperfusion in diabetic rats. Journal of Japanese Society of Nutrition and Food Science, 61, 119-127.

7) Li, Z. G., Britton, M., Sima, A. A. and Dunbar, J. C. (2004) Diabetes enhances apoptosis induced by cerebral ischemia. Life Sci., 76, 249-262.

8) Rizk, N. N., Rafols, J. and Dunbar, J. C. (2005) Cerebral ischemia induced apoptosis and necrosis in normal and diabetic rats. Brain Res., 1053, 1-9.

9) Li, P. A., Liu, G. J., He, Q. P., Floyd, R. A. and Siesjö, B. K. (1999) Production of hydroxyl free radical by brain tissues in hyperglycemic rats subjected to transient forebrain ischemia. Free Radic. Biol. Med., 27, 1033-1040.

10) Wei, J. and Quast, M. J. (1998) Effect of nitric oxide synthase inhibitor on a hyperglycemic rat model of reversible focal ischemia: detection of excitatory amino acids release and hydroxyl radical formation. Brain Res., 791, 146-156.

11) Kamalakkannan, N. and Stanely Mainzen Prince, P. (2006) Rutin improves the antioxidant status in streptozotocin-induced diabetic rat tissues. Mol. Cell. Biochem., 293, 211-219.

12) Montilla, P., Barcos, M., Munoz, M. C., Bujalance, I., Munoz-Castaneda, J. R. and Tunez, I. (2005) Red wine prevents brain oxidative stress and nephropathy in streptozotocin-induced diabetic rats. J. Biochem. Mol. Biol., 38, 539-544.

13) Kaleem, M., Asif, M., Ahmed, Q. U. and Bano, B. (2006) Antidiabetic and antioxidant activity of Annona squamosa extract in streptozotocin-induced diabetic rats. Singapore Med. J., 47, 670-675.

14) Attele, A. S., Zhou, Y. P., Xie, J. T., Wu, J. A., Zhang, L., Dey, L., Pugh, W., Rue, P. A., Polonsky, K. S. and Yuan, C. S. (2002) Antidiabetic effects of Panax ginseng berry extract and the identification of 
an effective component. Diabetes, 51, 1851-1858.

15) Kojo, S. (2004) Vitamin C: basic metabolism and its function as an index of oxidative stress. Curr. Med. Chem., 11, 1041-1064.

16) Will, J. C. and Byers, T. (1996) Does diabetes mellitus increase the requirement for vitamin C? Nutr. Rev., 54, 193-202.

17) Kashiba, M., Oka, J., Ichikawa, R., Kasahara, E., Inayama, T., Kageyama, A., Kageyama, H., Osaka, T., Umegaki, K., Matsumoto, A., Ishikawa, T., Nishikimi, M., Inoue, M. and Inoue, S. (2002) Impaired ascorbic acid metabolism in streptozotocininduced diabetic rats. Free Radic. Biol. Med., 33, 1221-1230.

18) Clarke, J., Snelling, J., Ioannides, C., Flatt, P. R. and Barnett, C. R. (1996) Effect of vitamin C supplementation on hepatic cytochrome P450 mixedfunction oxidase activity in streptozotocin-diabetic rats. Toxicol. Lett., 89, 249-256.

19) Çay, M., Naziroğlu, M., Simsek, H., Aydilek, N., Aksakal, M. and Demirci, M. (2001) Effects of intraperitoneally administered vitamin $\mathrm{C}$ on antioxidative defense mechanism in rats with diabetes induced by streptozotocin. Res. Exp. Med. (Berl.), 200, 205-213.

20) Sun, F., Iwaguchi, K., Shudo, R., Nagaki, Y., Tanaka, K., Ikeda, K., Tokumaru, S. and Kojo, S. (1999) Change in tissue concentrations of lipid hydroperoxides, vitamin $\mathrm{C}$ and vitamin $\mathrm{E}$ in rats with streptozotocin-induced diabetes. Clin. Sci. (Lond.), 96, 185-190.

21) Kashiba, M., Oka, J., Ichikawa, R., Kageyama, A., Inayama, T., Kageyama, H., Ishikawa, T., Nishikimi, M., Inoue M. and Inoue, S. (2000) Impaired reductive regeneration of ascorbic acid in the Goto-Kakizaki diabetic rat. Biochem. J., 351, 313318.

22) Huang, J., Agus, D. B., Winfree, C. J., Kiss, S., Mack, W. J., McTaggart, R. A., Choudhri, T. F., Kim, L. J., Mocco, J., Pinsky, D. J., Fox, W. D., Israel, R. J., Boyd, T. A., Golde, D. W. and Connolly, E. S., Jr. (2001) Dehydroascorbic acid, a blood-brain barrier transportable form of vitamin $\mathrm{C}$, mediates potent cerebroprotection in experimental stroke. Proc. Natl. Acad. Sci. U.S.A., 98, 1172011724.

23) Agus, D. B., Gambhir, S. S., Pardridge, W. M., Spielholz, C., Baselga, J., Vera, J. C. and Golde, D. W. (1997) Vitamin C crosses the blood-brain barrier in the oxidized form through the glucose transporters. J. Clin. Invest., 100, 2842-2848.

24) Carr, A. and Frei, B. (1999) Does vitamin C act as a pro-oxidant under physiological conditions? FASEB

$$
\text { J., 13, 1007-1024. }
$$

25) Song, J. H., Shin, S. H. and Ross, G. M. (2001) Oxidative stress induced by ascorbate causes neuronal damage in an in vitro system. Brain Res., 895, 6672.

26) Song, J. H., Shin, S. H., Wang, W. and Ross, G. M. (2001) Involvement of oxidative stress in ascorbateinduced proapoptotic death of PC12 cells. Exp. Neurol., 169, 425-437.

27) Trotti, R., Carratelli, M. and Barbieri, M. (2002) Performance and clinical application of a new, fast method for the detection of hydroperoxides in serum. Panminerva Med., 44, 37-40.

28) Ohkawa, H., Ohishi, N. and Yagi, K. (1979) Assay for lipid peroxides in animal tissues by thiobarbituric acid reaction. Anal. Biochem., 95, 351-358.

29) Tirkey, N., Kaur, G., Vij, G. and Chopra, K. (2005) Curcumin, a diferuloylmethane, attenuates cyclosporine-induced renal dysfunction and oxidative stress in rat kidneys. BMC Pharmacol., 15, 5, 15.

30) Shah, Z. A., Gilani, R. A., Sharma, P. and Vohora, S. B. (2005) Cerebroprotective effect of Korean ginseng tea against global and focal models of ischemia in rats. J. Ethnopharmacol., 101, 299-307.

31) Liu, K., Mori, S., Takahashi, H. K., Tomono, Y., Wake, H., Kanke, T., Sato, Y., Hiraga, N., Adachi, N., Yoshino, T. and Nishibori, M. (2007) Anti-high mobility group box 1 monoclonal antibody ameliorates brain infarction induced by transient ischemia in rats. FASEB J., 21, 3904-3916.

32) Nakada, Y., Yokoyama, O., Komatsu, K., Komada, K., Yotsuyanagi, S., Niikura, S., Nagasaka, Y. and Namiki, M. (2000) Effects of aniracetam on bladder overactivity in rats with cerebral infarction. J. Pharmacol. Exp. Ther., 293, 921-928.

33) Pari, L. and Latha, M. (2004) Protective role of Scoparia dulcis plant extract on brain antioxidant status and lipidperoxidation in STZ diabetic male Wistar rats. BMC Complement. Altern. Med., 4, 16.

34) Tsukaguchi, H., Tokui, T., Mackenzie, B., Berger, U. V., Chen, X. Z., Wang, Y., Brubaker, R. F. and Hediger, M. A. (1999) A family of mammalian $\mathrm{Na}^{+}$dependent L-ascorbic acid transporters. Nature, 399, 70-75.

35) Rumsey, S. C., Kwon, O., Xu, G. W., Burant, C. F., Simpson, I. and Levine, M. (1997) Glucose transporter isoforms GLUT1 and GLUT3 transport dehydroascorbic acid. J. Biol. Chem., 272, 18982-18989.

36) Minamizono, A., Tomi, M. and Hosoya, K. (2006) Inhibition of dehydroascorbic acid transport across the rat blood-retinal and -brain barriers in experimental diabetes. Biol. Pharm. Bull., 29, 2148-2150. 
37) Pardridge, W. M., Triguero, D. and Farrell, C. R. (1990) Downregulation of blood-brain barrier glucose transporter in experimental diabetes. Diabetes, 39, 1040-1044.

38) Simpson, I. A., Appel, N. M., Hokari, M., Oki, J., Holman, G. D., Maher, F., Koehler-Stec, E. M., Vannucci, S. J. and Smith, Q. R. (1999) Blood-brain barrier glucose transporter: effects of hypo- and hyperglycemia revisited. J. Neurochem., 72, 238-247.

39) Badr, G. A., Tang, J., Ismail-Beigi, F. and Kern, T. S. (2000) Diabetes downregulates GLUT1 expression in the retina and its microvessels but not in the cerebral cortex or its microvessels. Diabetes, 49, 10161021.

40) Huang, J., Agus, D. B., Winfree, C. J., Kiss, S., Mack, W. J., McTaggart, R. A., Choudhri, T. F., Kim, L. J., Mocco, J., Pinsky, D. J., Fox, W. D., Israel, R. J., Boyd, T. A., Golde, D. W. and Connolly, E. S., Jr. (2001) Dehydroascorbic acid, a blood-brain barrier transportable form of vitamin $\mathrm{C}$, mediates potent cerebroprotection in experimental stroke. Proc. Natl. Acad. Sci. U.S.A., 98, 1098910991.

41) Mack, W. J., Mocco, J., Ducruet, A. F., Laufer, I., King, R. G., Zhang, Y., Guo, W., Pinsky, D. J. and Connolly, E. S., Jr. (2006) A cerebroprotective dose of intravenous citrate/sorbitol-stabilized dehydroascorbic acid is correlated with increased cerebral ascorbic acid and inhibited lipid peroxidation after murine reperfused stroke. Neurosurgery, 59, 383-388.

42) Bémeur, C., Ste-Marie, L., Desjardins, P., Vachon, L., Butterworth, R. F., Hazell, A. S. and Montgomery, J. (2005) Dehydroascorbic acid normalizes several markers of oxidative stress and inflammation in acute hyperglycemic focal cerebral ischemia in the rat. Neurochem. Int., 46, 399-407.

43) Kim, E. J., Won, R., Sohn, J. H., Chung, M. A., Nam, T. S., Lee, H. J. and Lee, B. H. (2008) Anti-oxidant effect of ascorbic and dehydroascorbic acids in hippocampal slice culture. Biochem. Biophys. Res. Commun., 366, 8-14.

44) Nualart, F. J., Rivas, C. I., Montecinos, V. P., Godoy, A. S., Guaiquil, V. H., Golde, D. W. and Vera, J. C. (2003) Recycling of vitamin C by a bystander effect. J. Biol. Chem., 278, 10128-10133.

45) Kamada, H., Yu, F., Nito, C. and Chan, P. H. (2007) Influence of hyperglycemia on oxidative stress and matrix metalloproteinase-9 activation after focal cerebral ischemia/reperfusion in rats: relation to blood-brain barrier dysfunction. Stroke, 38, 1044-1049. 